\title{
The Anatomy of the Mesolimbic Reward System: A Link between Personality and the Placebo Analgesic Response
}

\author{
Petra Schweinhardt, ${ }^{1}$ David A. Seminowicz, ${ }^{1}$ Erik Jaeger, ${ }^{1}$ Gary H. Duncan, ${ }^{2}$ and M. Catherine Bushnell ${ }^{1}$ \\ ${ }^{1}$ Alan Edwards Centre for Research on Pain, Faculty of Dentistry, McGill University, Montreal, Quebec, Canada H3A2B2, and ²Département de \\ Stomatologie, Université de Montréal, Montréal, Québec, Canada H3T 1J4
}

The anticipation of clinical benefit, a crucial component of placebo analgesia, has been suggested to be a special case of reward anticipation. Since reward processing is closely linked to the ventral striatum and the neurotransmitter dopamine, we examined the relationships between brain gray matter, placebo analgesic response, and personality traits associated with dopaminergic neurotransmission. We report that dopamine-related traits predict a substantial portion of the pain relief an individual gains from a sham treatment. Voxelbased morphometry of magnetic resonance images shows that the magnitude of placebo analgesia is related to gray matter density (GMD) in several brain regions, including the ventral striatum, insula, and prefrontal cortex. Similarly, GMD in ventral striatum and prefrontal cortex is related to dopamine-related personality traits. Our findings highlight the relationship between placebo and reward and potentially offer ways of identifying subjects who are likely to show large placebo analgesic responses.

\section{Introduction}

The neurobiology behind the placebo effect is perhaps best known for placebo analgesia, i.e., pain relief caused by administration of a sham treatment. Several brain systems have been implicated in mediating placebo analgesic responses. The dorsolateral prefrontal cortex (DLPFC) seems particularly concerned with the anticipation of pain relief (Wager et al., 2004), and the rostral anterior cingulate cortex (rACC) possibly engages descending antinociceptive systems (Petrovic et al., 2002; Bingel et al., 2006; Wager et al., 2007). The release of endogenous opioids (Zubieta et al., 2005; Scott et al., 2008) as well as dopamine (Scott et al., 2008) during placebo analgesia has been shown to occur in several brain regions, including the ventral striatum, which is part of the mesolimbic reward system. Interestingly, dopamine in the ventral striatum is not only released during the actual experience of placebo analgesia but also during the anticipation of placeboinduced pain relief (Scott et al., 2007). This supports a recent theory that conceptualizes the anticipation of clinical benefit, crucial for the placebo response, as a special case of reward anticipation (de la Fuente-Fernández et al., 2001). Reward theory distinguishes between the anticipation of reward, closely linked to the motivation to obtain reward, and the actual hedonic experience of reward ("wanting" vs "liking") (Berridge, 1996). Whereas the "liking" component is associated with striatal opioids (Peciña and Berridge, 2000), "wanting" seems to be related to dopaminergic neurotransmission in the ventral striatum (Wyvell and

\footnotetext{
Received Nov. 10, 2008; revised March 9, 2009; accepted March 16, 2009.

We acknowledge the Canadian Institutes of Health Research for financial support and an Astra Zeneca/Alan Edwards Centre for Research on Pain postdoctoral fellowship to P.S.

Correspondence should be addressed to Petra Schweinhardt, Alan Edwards Centre for Research on Pain, McGill University, Room M/19, Strathcona Anatomy and Dentistry Building, 3640 University Street, Montreal, Quebec, Canada H3A 2B2. E-mail: petra.schweinhardt@mcgill.ca.

D01:10.1523/JNEUROSCI.5634-08.2009

Copyright $\odot 2009$ Society for Neuroscience $\quad 0270-6474 / 09 / 294882-06 \$ 15.00 / 0$
}

Berridge, 2000). Individuals differ with respect to their sensitivity to reward and reward-predicting cues (Shoaib et al., 1995; Cohen et al., 2005; Beaver et al., 2006), and in particular, personality traits linked to dopaminergic neurotransmission are associated with reward sensitivity (Yacubian et al., 2007). In the case of placebo analgesia, the magnitude of opioid as well as dopamine release in the ventral striatum is related to the amount of pain relief an individual experiences (Zubieta et al., 2005; Scott et al., $2007,2008)$. For dopamine, this is remarkable because, with respect to reward, a perceptual change in outcome (i.e., the actual experience of reward) seems to be more closely related to opioidergic than to dopaminergic neurotransmission. However, there is evidence that $\mathrm{D}_{2}$-receptor activation can induce analgesia without depending on opioids (Drago et al., 1984; Shimizu et al., 2004).

Magnetic resonance imaging (MRI)-based measures of gray matter have been shown to be related to brain function, both in health and disease (e.g., Peinemann et al., 2005; Newman et al., 2007), possibly because they partly reflect the number and size of neurons and the complexity of their synaptic connections. Likewise, individual anatomical differences within dopaminergic pathways have been linked to significant behavioral effects, including variation of personality traits (Depue and Collins, 1999). Since the ventral striatum plays such a prominent role for reward processing and placebo analgesia, we hypothesized that the magnitude of pain relief an individual gains from a sham treatment would be related to gray matter in this region. We further hypothesized that dopamine-related personality traits would partially predict the magnitude of the placebo analgesic responses in a given individual and would be related to gray matter in the ventral striatum.

\section{Materials and Methods \\ Subjects}

Twenty-two healthy males (mean age: 22.2 years, SD: 4.0 years) were investigated. Subjects were carefully screened for any medical condition, including neurological and psychiatric problems. All procedures were 
approved by the local ethics committee, and written informed consent was obtained from all participants.

\section{Experimental procedures}

General procedure. To test our hypotheses, we established the magnitude of the placebo analgesic response using a within-subject psychophysical paradigm. A high resolution MRI scan was obtained for every subject. Gray matter density (GMD) was examined using voxel-based morphometry (VBM). Personality traits related to dopaminergic neurotransmission were assessed using self-report questionnaires.

Subjects underwent three sessions: one manipulation session, one testing session, and one MRI/questionnaire session. The manipulation and the testing sessions were 1 or $2 \mathrm{~d}$ apart, the MRI was performed between 1 and 2 weeks later.

Manipulation session. A conditioning-like procedure was used to enhance the effectiveness of the placebo treatment. This procedure has been successfully used in previous studies of placebo analgesia (Price et al., 1999; Wager et al., 2004; Bingel et al., 2006) (for details, see supplemental material, available at www.jneurosci.org).

Testing session. The study followed a counterbalanced, cross-over design: half of the subjects received a painful intramuscular infusion of hypertonic saline paired with a control cream first, followed by an identical hypertonic saline infusion paired with a cream that subjects were made to believe was analgesic. In fact, the analgesic cream was identical to the control cream. The order was reversed for the other half of the subjects. Details of the hypertonic saline infusions can be found in the supplemental material (available at www.jneurosci.org).

Psychophysical ratings. Throughout the infusions, subjects rated the intensity (0: "no sensation," 100: "pain threshold," 200: "most intense pain tolerable") and the pleasantness/unpleasantness ( -100 : "extremely unpleasant," 0: neutral, 100: "extremely pleasant") of the sensation every $1.5 \mathrm{~min}$ using visual analogous scales (VASs). These scales are sensitive to detect changes in pain perception induced by psychological manipulation (Villemure et al., 2003). Pain intensity and unpleasantness were analyzed as maxima and area under the curve (AUC). Immediately after each infusion, subjects provided mood and anxiety ratings on VASs (mood scale: -100: "extremely bad mood," 0: "neutral," 100: extremely good mood"; anxiety scale: -100 : "extremely anxious, “ 0 : ”neutral, “ 100 : "extremely calm").

Assessment of personality traits. Using self-report questionnaires, we assessed the following traits, which have a suggested relationship to dopaminergic neurotransmission: novelty seeking (Temperament and Character Inventory, TCI, Cloninger, 1987), harm avoidance (inversely related to dopamine; TCI), behavioral drive [subscale of the Behavioral Appetitive System (BAS) scale (Carver and White, 1994)], fun seeking (BAS), and reward responsiveness (BAS) (for studies providing evidence for relationship to dopamine, see supplemental material, available at www.jneurosci.org). Principal component analysis was used for data reduction. All variables loaded on to one component (harm avoidance loaded negatively, in accordance with previous literature showing an inverse relationship with dopaminergic neurotransmission) (supplemental Table S1, available at www.jneurosci.org as supplemental material), confirming that they are measuring related traits. Each individual's factor score of this component was used for subsequent analyses as the "dopamine-related trait variable."

MRI. Imaging was performed using a $1.5 \mathrm{~T}$ Siemens Vision scanner (Siemens Medical Systems) with a standard head-coil. High-resolution T1-weighted anatomical scans were acquired (TR, $22 \mathrm{~ms}$; TE, $9.2 \mathrm{~ms}$; flip angle, $30^{\circ}$; FOV, $256 \mathrm{~mm}$; voxel size $1 \times 1 \times 1 \mathrm{~mm}$ ).

\section{Data analysis}

Psychophysical data. Ratings of pain intensity, pain unpleasantness, anxiety/calmness, and mood were compared between conditions using onesided $t$ tests $(p<0.05)$. For each subject, the percentage difference (AUC) for pain intensity between the placebo condition and the control condition was calculated to yield a measure of the individual's placebo analgesic response. The placebo analgesic response was used for as regressor in the general linear model (GLM) of the VBM analysis as well as to examine a potential relationship between placebo analgesic response
Table 1. Psychophysical results

\begin{tabular}{lccl}
\hline & Placebo mean (SD) & Control mean (SD) & $p$ value $^{a}$ \\
\hline Maximum pain intensity & $157(18.9)$ & $164(14.7)$ & 0.015 \\
Maximum unpleasant- & & & \\
$\quad$ ness & $-58.5(19.1)$ & $-63(16.6)$ & 0.059 \\
Anxiety/calmness & $11.7(42.9)$ & $1.4(31.8)$ & 0.07 \\
Mood & $17.7(39.6)$ & $18.5(37.5)$ & 0.45 \\
\hline
\end{tabular}

The maximum pain subjects experienced during the hypertonic saline infusion was significantly lower in the placebo condition than in the control condition across the whole group.

${ }^{a}$ One-sided $t$ test.

and the dopamine-related trait variable (Pearson's correlation coefficient, $p<0.05$ for two-sided tests). Similarly, the change in pain unpleasantness (percentage difference between conditions, AUC) was correlated with the dopamine-related trait variable. Last, we calculated Pearson's correlation coefficient ( $p<0.05$ for two-sided tests) between the placebo analgesic response and changes in mood and anxiety/calmness.

VBM. The VBM methods used were identical to those described previously (Kuchinad et al., 2007; Schweinhardt et al., 2008). Briefly, each image underwent automated correction for intensity nonuniformity and intensity standardization. The MRI volumes were spatially normalized to a T1-weighted template in stereotaxic space (the MNI/ICBM 152 standard) using linear transformations. Brain tissue was classified into gray matter, white matter, CSF, and background, and the skull, brainstem, cerebellum, and dura were removed from further analysis. The smoothed images (Gaussian kernel of $10 \mathrm{~mm}$ full width at half maximum) were used as three-dimensional maps for GMD, measured from the intensity of the image within each voxel (for details, see supplemental material, available at www.jneurosci.org).

Correlations between GMD and placebo analgesic response. A univariate GLM was used to examine whether GMD depended on the individuals' placebo analgesic response (explanatory variable), which calculated a $t$-statistic for each voxel. Since none of the regions that have been implicated in placebo analgesia is located in the posterior part of the brain (except the brainstem, which is excluded by default from the VBM analysis used here), we restricted our analysis to gray matter anterior to the precentral gyrus ( $y=-15.5$, MNI coordinates). This volume, which corresponds to $46 \%$ of the total brain gray matter, includes the frontal cortex anterior to the precentral gyrus, the insula, the basal ganglia, and part of the thalamus. Statistical inference was based on cluster size [spatial extent of contiguous voxels above a voxel-based threshold of $p<$ 0.05 , corrected for spatial extent at $p<0.05$ across the volume of interest $\left.\left(759 \mathrm{~cm}^{3}\right)\right]$.

Correlations between GMD and dopamine-related trait variable. The same analysis as for the placebo analgesic response was performed using the dopamine-related trait variable as explanatory variable in the GLM.

Partial correlations. To examine whether correlations between gray matter density and placebo analgesic response can be explained by a relationship with the dopamine-related trait variable and vice versa, we computed partial correlations $(p<0.05)$ for all clusters that were significant in the GLM analyses. Mean GMD in a cluster served as dependent variable, placebo analgesic response or dopamine-related trait variable as independent variable, controlled for the respective other variable (covariate of no interest).

\section{Results}

\section{Placebo analgesic response}

The hypertonic saline elicited significantly lower pain intensity ratings in the placebo condition compared with the control condition across the whole group (Table 1). Unpleasantness and anxiety/calmness ratings showed a trend to differ between placebo and control condition (Table 1). The changes in mood and calmness/anxiety between the placebo and the control condition did not correlate with the placebo analgesic response $(r=-0.26$, $p=0.24$ and $r=-0.08, p=0.7)$. 
Table 2. Gray matter density correlated with placebo analgesia or dopamine-related trait variable

\begin{tabular}{|c|c|c|c|c|c|c|c|c|}
\hline \multirow[b]{2}{*}{ Location } & \multicolumn{3}{|c|}{ MNI coordinates (peak) } & \multirow[b]{2}{*}{$t$ value (peak) } & \multirow[b]{2}{*}{ Cluster extent $\left(\mathrm{mm}^{3}\right)$} & \multirow[b]{2}{*}{$p$ value (cluster extent) } & \multirow[b]{2}{*}{$r$ (p value) } & \multirow[b]{2}{*}{$r_{\mathrm{p}}(p$ value $)$} \\
\hline & $x$ & $y$ & $Z$ & & & & & \\
\hline \multicolumn{9}{|c|}{$\begin{array}{c}\text { Correlation with magnitude } \\
\text { of placebo analgesia }\end{array}$} \\
\hline Insula/TC & -47 & -2 & -7 & 3.7 & 6300 & 0.002 & $0.67(0.001)$ & $0.64(0.002)$ \\
\hline \multirow[t]{2}{*}{ Ventral striatum } & -13 & 11 & -12 & 3.4 & 2628 & 0.029 & $0.61(0.003)$ & $0.62(0.003)$ \\
\hline & 12 & 16 & -5 & 2.9 & 2280 & 0.039 & $0.54(0.01)$ & $0.35(0.12)$ \\
\hline Middle frontal gyrus & 28 & 10 & 47 & 4.4 & 2442 & 0.034 & $0.71(<0.001)$ & $0.71(<0.001)$ \\
\hline \multicolumn{9}{|c|}{$\begin{array}{l}\text { Correlation with dopamine- } \\
\text { related trait variable }\end{array}$} \\
\hline $\mathrm{MPFC} / \mathrm{rACC}$ & 16 & 54 & 6 & 2.6 & 1106 & $0.13^{a}$ & & \\
\hline DLPFC & 45 & 45 & 26 & 4.0 & 6592 & 0.002 & $0.65(0.001)$ & $0.68(0.001)$ \\
\hline Ventral striatum & 13 & 18 & -1 & 3.1 & 3319 & 0.017 & $0.57(0.006)$ & $0.42(0.061)$ \\
\hline Operculum/TC & 54 & 11 & 1 & 3.3 & 2455 & 0.033 & $0.53(0.01)$ & $0.54(0.01)$ \\
\hline
\end{tabular}

MNI, Montreal Neurological Institute; TC, temporal cortex; MPFC, medial prefrontal cortex; rACC, rostral anterior cingulate cortex; DLPFC, dorsolateral prefrontal cortex. $r$ is Pearson's correlation coefficient; $r_{\mathrm{p}}$ refers to the partial correlation coefficient between mean GMD in the cluster and placebo analgesic response (top) or dopamine-related trait variable (bottom) [controlled for dopamine-related trait variable (top) or placebo analgesic response (bottom)].

${ }^{a}$ Clusters in the posterior insula as well as MPFC/rACC are not statistically significant but were included for interest.

\section{The placebo analgesic response correlates with dopamine- related personality traits}

The dopamine-related trait variable explained $\sim 30 \%$ of the variance of the placebo-induced change in pain intensity $\left(r=0.58, r^{2}\right.$ $=0.34, r^{2}$ adjusted $\left.=0.31, F_{(1,21)}=10.3, p=0.004\right)$ and pain unpleasantness $\left(r=0.57, r^{2}=0.32, r^{2}\right.$ adjusted $=0.29, F_{(1,21)}=9.5$, $p=0.006)$.

\section{Gray matter correlates with the magnitude of}

\section{placebo analgesia}

In support of our hypothesis, the individual's placebo analgesic response correlated with GMD bilaterally in the ventral striatum (Table 2, Fig. 1). In addition, GMD in a cluster including insula and temporal cortex, as well as in the medial frontal gyrus correlated significantly with the placebo analgesic response (Table 2, Fig. 1). To present a more complete picture, Figure 1 and Table 2 also depict clusters that did not reach statistical significance, such as rACC and medial prefrontal cortex.

\section{Gray matter correlates with the dopamine-related personality traits}

GMD in three clusters correlated with the dopamine-related trait variable, located in the right ventral striatum, DLPFC, and operculum/temporal cortex (Table 2, Fig. 2).

\section{Partial correlations}

In most regions, there were unique relationships between GMD and either placebo analgesic response or dopamine-related trait variable, i.e., the original correlations were not influenced by including the covariate of no interest (Table 2). In one single area, namely in the right ventral striatum, the present study cannot distinguish whether GMD is uniquely dependent on either the analgesic response or the dopamine-related traits since correlations of either independent variable depended on the respective other variable (Table 2). Further study is warranted to shed light on whether placebo analgesic responses or dopamine-related traits mediate the relationship with GMD in the right ventral striatum.

\section{Discussion}

Here, we present two novel findings. First, personality traits that have previously been linked to dopaminergic neurotransmission contribute to an individual's placebo analgesic response. Second, GMD in the ventral striatum and other brain regions is related to the magnitude of the placebo analgesia. In addition, dopaminerelated personality traits were related to gray matter in the ventral striatum, DLPFC, and operculum/temporal cortex. This is in accordance with a previous study, which demonstrated a relationship between GMD in the DLPFC and "novelty seeking" (Iidaka et al., 2006), and a more recent study, which described an inverse correlation between GMD in the anterior caudate nucleus/ventral striatum and anhedonia, emphasizing the role of this region in reward processing (Harvey et al., 2007). Our results demonstrate a clear relationship between dopamine-related personality traits, ventral striatum gray matter, and placebo analgesic response.

Results of the present study contrast with those of the existing literature that had largely failed to confirm a relationship between personality and placebo response (Turner et al., 1994; Hoffman et al., 2005) [but see De Pascalis et al. (2002) for a relationship between suggestibility and placebo analgesia]. However, to the best of our knowledge, no previous work tested the personality traits examined here for a potential relationship to placebo analgesia. A combination of dopamine-related traits accounted for $\sim 30 \%$ of the variance in the placebo analgesic response. A post hoc analysis examining the individual traits showed that novelty seeking, behavioral drive, and fun seeking correlated significantly with the placebo analgesic response, whereas harm avoidance and reward responsiveness did not (supplemental Table S2, available at www.jneurosci.org as supplemental material). Since several of the individual traits correlated with the placebo analgesic response, the positive finding in the present study cannot simply be explained by the fact that we took an approach that has not been taken by earlier studies, i.e., using a composite measure of several personality traits. Interestingly, a previous study found a similar proportion of placebo analgesia to be explained by ventral striatum activation during anticipation of monetary reward (Scott et al., 2007). Although that study did not specifically investigate personality traits, it has previously been shown that dopaminerelated traits predict the ventral striatum response to reward (Beaver et al., 2006). Hence, ventral striatum anatomy and function might be considered endophenotypes of dopamine-related personality traits that indicate susceptibility to placebo analgesia.

It is important to emphasize that other pathways, which are independent of dopamine, have been shown to be involved in placebo analgesia. For example, the ventral striatum is also an important site of opioidergic neurotransmission, and conse- 

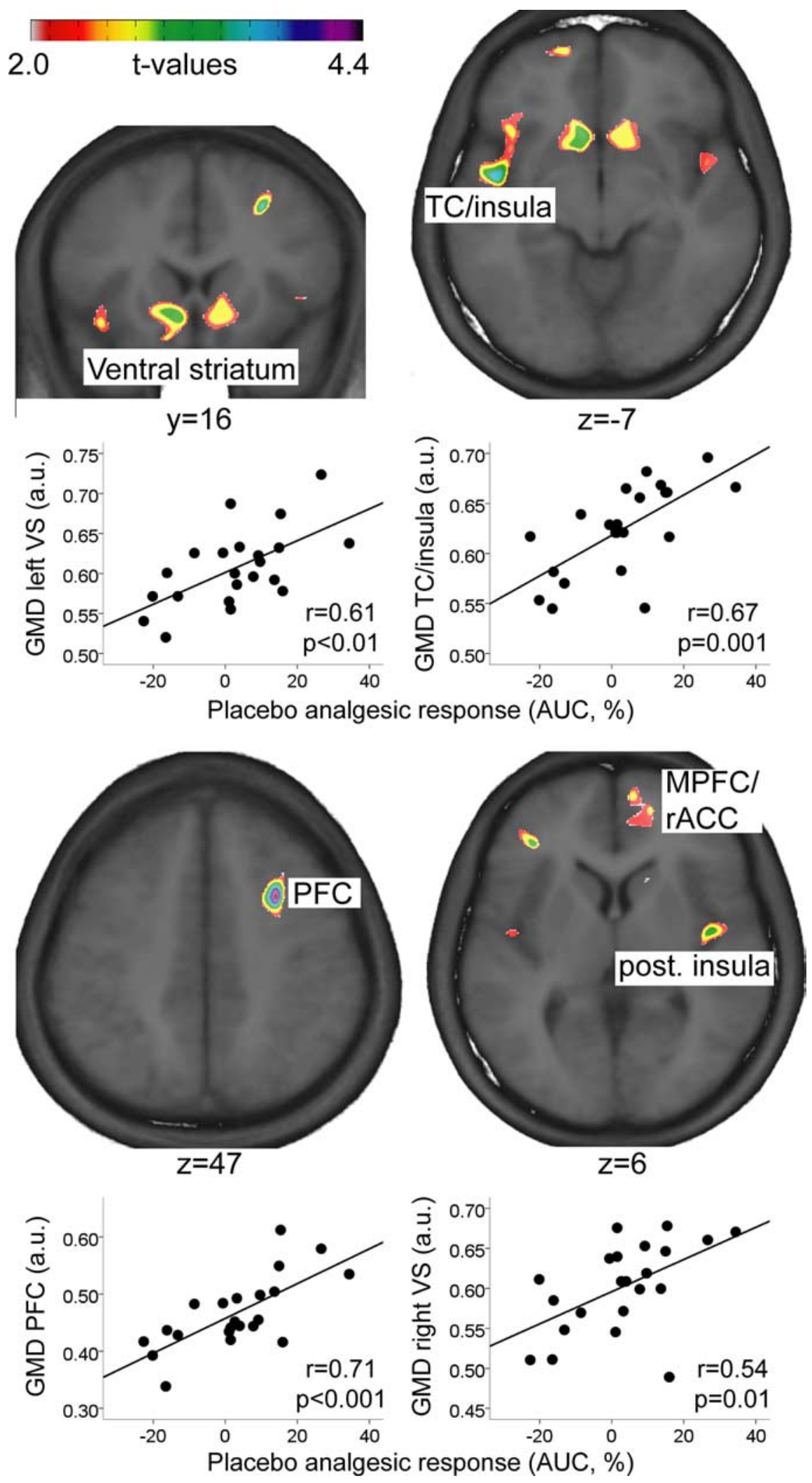

Figure 1. Areas in which the magnitude of placebo analgesia correlated with gray matter density. Please note that the clusters in the MPFC/rACC and posterior insula did not reach statistical significance. Scatter plots depict the relationships between mean GMD in the significant clusters and placebo analgesic response (positive values correspond to decreased pain ratings in placebo compared with control condition). Images in neurological convention. TC, Temporal cortex; (M)PFC, (medial) prefrontal cortex; rACC, rostral anterior cingulate cortex; a.u., arbitrary units; AUC, area under the curve; GMD, gray matter density). quently, the link between placebo analgesia and ventral striatum might be accounted for partly by interindividual differences in opioidergic function. Schreckenberger et al. (2008) showed that opioid receptor binding potentials in the ventral striatum correlate with the trait of reward dependence. In contrast, they did not find a relationship between opioids and novelty seeking. We therefore infer that our results involving the ventral striatum are likely to be related to the dopamine system.

There are several possible ways how dopamine might contribute to placeboinduced analgesia. First, dopamine plays a role in inducing a positive affective state (Oswald et al., 2005), which could lead to secondary pain relief (Villemure et al., 2003). This possibility is not supported by our data, which did not reveal a relationship between changes in mood or anxiety and placebo analgesic response. A second possibility is that placebo-induced pain relief is partly a direct effect of dopamine since there is accumulating evidence that $\mathrm{D}_{2}$-receptor agonism inhibits pain (Shimizu et al., 2004). Last, within the general framework of reward, dopamine is considered crucial for the motivational drive to obtain a rewarding stimulus (or clinical benefit) whereas the release of endogenous opioids is thought to mediate the pleasure associated with the obtained reward (or pain relief) (Berridge, 1996). Hence, it seems likely that dopamine could contribute to opioid-mediated analgesia by increasing the motivation to obtain clinical benefit, although it is not yet fully understood how dopaminergic pathways modulate opioid release.

In addition to the ventral striatum, GMD in several other brain regions correlated with either the magnitude of the placebo analgesic response or with the dopamine-related trait variable. All the regions identified in the present study have been functionally implicated in placebo analgesia by activation (Petrovic et al., 2002; Wager et al., 2004; Bingel et al., 2006) or dopamine-/opioid-receptor binding studies (Zubieta et al., 2005; Scott et al., 2007, 2008). The lateral prefrontal cortex, and especially the DLPFC, seems to play a crucial role in the anticipation of placebo analgesia (Wager et al., 2004), in line with the observation that some DLPFC neurons encode reward expectancy (Wallis and Miller, 2003). There is ample evidence for functional interactions between DLPFC and striatum (e.g., Kröner et al., 2007). Of importance for the anatomical findings of the present study is 
the observation that the concentration of DLPFC $N$-acetyl-aspartate, which might be considered a neurochemical homolog of GMD, predicts striatal $\mathrm{D}_{2}$-receptor availability (Bertolino et al., 1999). The DLPFC uses reward information to generate motivational context and control behavior (Wallis and Miller, 2003). With respect to pain, the lateral prefrontal cortex has been shown to be important for endogenous pain control in a variety of different circumstances (Lorenz et al., 2003; Wiech et al., 2006, 2008).

Gray matter in the insula correlated with the magnitude of placebo analgesia and a cluster in the frontal operculum/ temporal cortex, adjacent to the insula, correlated with dopamine-related personality traits. The insula does not only exhibit a relationship between dopaminergic tone and the personality trait of novelty seeking (Suhara et al., 2001) but has also been shown in rat studies to play a role in pain modulation, including descending networks (Burkey et al., 1999). In the rat, dopamine and GABA appear to be key neurotransmitters in the function of this cortical region, at least in the anterior part (Ohara et al., 2003). Animal studies suggest that part of the dopaminergic modulation of the anterior insula occurs through GABAergic interneurons projecting to the ventral striatum (Ohara et al., 2003).

\section{Limitations}

First, due to the correlative nature of the study, it does not provide information regarding causation or directionality of the relationships between the variables GMD in right ventral striatum, placebo analgesic response, and dopamine-related trait variable. Second, we cannot definitively ascertain a dopaminergic link between these three variables since we did not measure dopamine. To test this association directly, positron emission tomography studies of dopamine binding potential and pharmacological studies that enhance or impair dopaminergic neurotransmission are needed. Thirdly, our interpretation might only apply to male subjects, since the dopaminergic system differs between genders, also with respect to expectation-induced dopamine release (Haltia et al., 2008). Last, the ventral striatum has also been implicated in the processing of aversive events, including pain (Becerra et al., 2001). Consequently, the observed link between striatum and placebo could be due to aversive anticipatory signaling, rather than to an appetitive pathway associated with the reward system. We cannot clearly differentiate these two explanations in this study, and indeed, they might be closely connected: anticipation of reward (as pain relief) is essentially dependent on the degree of anticipated pain; i.e., higher levels of expected pain would be correlated with a greater reward for mounting a stronger placebo response.

In conclusion, this study shows that the magnitude of placebo analgesia and dopamine-related personality traits are related to brain gray matter, in particular in the ventral striatum. This further emphasizes the important role of mesolimbic dopaminergic pathways for placebo-induced pain relief and supports the idea that the placebo response is related to reward processing.

\section{References}

Beaver JD, Lawrence AD, van Ditzhuijzen J, Davis MH, Woods A, Calder AJ (2006) Individual differences in reward drive predict neural responses to images of food. J Neurosci 26:5160-5166.

Becerra L, Breiter HC, Wise R, Gonzalez RG, Borsook D (2001) Reward circuitry activation by noxious thermal stimuli. Neuron 32:927-946.

Berridge KC (1996) Food reward: brain substrates of wanting and liking. Neurosci Biobehav Rev 20:1-25.

Bertolino A, Knable MB, Saunders RC, Callicott JH, Kolachana B, Mattay VS, Bachevalier J, Frank JA, Egan M, Weinberger DR (1999) The relationship between dorsolateral prefrontal $N$-acetylaspartate measures and striatal dopamine activity in schizophrenia. Biol Psychiatry 45:660-667.

Bingel U, Lorenz J, Schoell E, Weiller C, Büchel C (2006) Mechanisms of placebo analgesia: rACC recruitment of a subcortical antinociceptive network. Pain 120:8-15.

Burkey AR, Carstens E, Jasmin L (1999) Dopamine reuptake inhibition in the rostral agranular insular cortex produces antinociception. J Neurosci 19:4169-4179.

Carver CS, White TL (1994) Behavioral inhibition, behavioral activation, and affective responses to impending reward and punishment: the BIS/ BAS scales. J Pers Soc Psychol 67:319-333.

Cloninger CR (1987) A systematic method for clinical description and classification of personality variants. A proposal. Arch Gen Psychiatry 44:573-588.

Cohen MX, Young J, Baek JM, Kessler C, Ranganath C (2005) Individual differences in extraversion and dopamine genetics predict neural reward responses. Brain Res Cogn Brain Res 25:851-861.

de la Fuente-Fernández R, Ruth TJ, Sossi V, Schulzer M, Calne DB, Stoessl AJ (2001) Expectation and dopamine release: mechanism of the placebo effect in Parkinson's disease. Science 293:1164-1166.

De Pascalis V, Chiaradia C, Carotenuto E (2002) The contribution of suggestibility and expectation to placebo analgesia phenomenon in an experimental setting. Pain 96:393-402.

Depue RA, Collins PF (1999) Neurobiology of the structure of personality: dopamine, facilitation of incentive motivation, and extraversion. Behav Brain Sci 22:491-517; discussion 518-469.

Drago F, Caccamo G, Continella G, Scapagnini U (1984) Amphetamine- 
induced analgesia does not involve brain opioids. Eur J Pharmacol 101:267-269.

Haltia LT, Rinne JO, Helin S, Parkkola R, Någren K, Kaasinen V (2008) Effects of intravenous placebo with glucose expectation on human basal ganglia dopaminergic function. Synapse 62:682-688.

Harvey PO, Pruessner J, Czechowska Y, Lepage M (2007) Individual differences in trait anhedonia: a structural and functional magnetic resonance imaging study in non-clinical subjects. Mol Psychiatry 12:703, 767-775.

Hoffman GA, Harrington A, Fields HL (2005) Pain and the placebo: what we have learned. Perspect Biol Med 48:248-265.

Iidaka T, Matsumoto A, Ozaki N, Suzuki T, Iwata N, Yamamoto Y, Okada T, Sadato N (2006) Volume of left amygdala subregion predicted temperamental trait of harm avoidance in female young subjects. A voxel-based morphometry study. Brain Res 1125:85-93.

Kröner S, Krimer LS, Lewis DA, Barrionuevo G (2007) Dopamine increases inhibition in the monkey dorsolateral prefrontal cortex through cell typespecific modulation of interneurons. Cereb Cortex 17:1020-1032.

Kuchinad A, Schweinhardt P, Seminowicz DA, Wood PB, Chizh BA, Bushnell MC (2007) Accelerated brain gray matter loss in fibromyalgia patients: premature aging of the brain? J Neurosci 27:4004-4007.

Lorenz J, Minoshima S, Casey KL (2003) Keeping pain out of mind: the role of the dorsolateral prefrontal cortex in pain modulation. Brain 126:1079-1091.

Newman LM, Trivedi MA, Bendlin BB, Ries ML, Johnson SC (2007) The relationship between gray matter morphometry and neuropsychological performance in a large sample of cognitively healthy adults. Brain Imaging Behav 1:3-10.

Ohara PT, Granato A, Moallem TM, Wang BR, Tillet Y, Jasmin L (2003) Dopaminergic input to GABAergic neurons in the rostral agranular insular cortex of the rat. J Neurocytol 32:131-141.

Oswald LM, Wong DF, McCaul M, Zhou Y, Kuwabara H, Choi L, Brasic J, Wand GS (2005) Relationships among ventral striatal dopamine release, cortisol secretion, and subjective responses to amphetamine. Neuropsychopharmacology 30:821-832.

Peciña S, Berridge KC (2000) Opioid site in nucleus accumbens shell mediates eating and hedonic 'liking' for food: map based on microinjection Fos plumes. Brain Res 863:71-86.

Peinemann A, Schuller S, Pohl C, Jahn T, Weindl A, Kassubek J (2005) Executive dysfunction in early stages of Huntington's disease is associated with striatal and insular atrophy: a neuropsychological and voxel-based morphometric study. J Neurol Sci 239:11-19.

Petrovic P, Kalso E, Petersson KM, Ingvar M (2002) Placebo and opioid analgesia-imaging a shared neuronal network. Science 295:1737-1740.

Price DD, Milling LS, Kirsch I, Duff A, Montgomery GH, Nicholls SS (1999) An analysis of factors that contribute to the magnitude of placebo analgesia in an experimental paradigm. Pain 83:147-156.

Schreckenberger M, Klega A, Gründer G, Buchholz HG, Scheurich A, Schirrmacher R, Schirrmacher E, Müller C, Henriksen G, Bartenstein P (2008)
Opioid receptor PET reveals the psychobiologic correlates of reward processing. J Nucl Med 49:1257-1261.

Schweinhardt P, Kuchinad A, Pukall CF, Bushnell MC (2008) Increased gray matter density in young women with chronic vulvar pain. Pain 140:411-419.

Scott DJ, Stohler CS, Egnatuk CM, Wang H, Koeppe RA, Zubieta JK (2007) Individual differences in reward responding explain placebo-induced expectations and effects. Neuron 55:325-336.

Scott DJ, Stohler CS, Egnatuk CM, Wang H, Koeppe RA, Zubieta JK (2008) Placebo and nocebo effects are defined by opposite opioid and dopaminergic responses. Arch Gen Psychiatry 65:220-231.

Shimizu T, Iwata S, Morioka H, Masuyama T, Fukuda T, Nomoto M (2004) Antinociceptive mechanism of L-DOPA. Pain 110:246-249.

Shoaib M, Spanagel R, Stohr T, Shippenberg TS (1995) Strain differences in the rewarding and dopamine-releasing effects of morphine in rats. Psychopharmacology (Berl) 117:240-247.

Suhara T, Yasuno F, Sudo Y, Yamamoto M, Inoue M, Okubo Y, Suzuki K (2001) Dopamine D2 receptors in the insular cortex and the personality trait of novelty seeking. Neuroimage 13:891-895.

Turner JA, Deyo RA, Loeser JD, Von Korff M, Fordyce WE (1994) The importance of placebo effects in pain treatment and research. JAMA 271:1609-1614.

Villemure C, Slotnick BM, Bushnell MC (2003) Effects of odors on pain perception: deciphering the roles of emotion and attention. Pain 106:101-108.

Wager TD, Rilling JK, Smith EE, Sokolik A, Casey KL, Davidson RJ, Kosslyn SM, Rose RM, Cohen JD (2004) Placebo-induced changes in FMRI in the anticipation and experience of pain. Science 303:1162-1167.

Wager TD, Scott DJ, Zubieta JK (2007) Placebo effects on human muopioid activity during pain. Proc Natl Acad Sci U S A 104:11056-11061.

Wallis JD, Miller EK (2003) Neuronal activity in primate dorsolateral and orbital prefrontal cortex during performance of a reward preference task. Eur J Neurosci 18:2069-2081.

Wiech K, Kalisch R, Weiskopf N, Pleger B, Stephan KE, Dolan RJ (2006) Anterolateral prefrontal cortex mediates the analgesic effect of expected and perceived control over pain. J Neurosci 26:11501-11509.

Wiech K, Farias M, Kahane G, Shackel N, Tiede W, Tracey I (2008) An fMRI study measuring analgesia enhanced by religion as a belief system. Pain 139:467-476

Wyvell CL, Berridge KC (2000) Intra-accumbens amphetamine increases the conditioned incentive salience of sucrose reward: enhancement of reward "wanting" without enhanced "liking" or response reinforcement. J Neurosci 20:8122-8130.

Yacubian J, Sommer T, Schroeder K, Gläscher J, Kalisch R, Leuenberger B, Braus DF, Büchel C (2007) Gene-gene interaction associated with neural reward sensitivity. Proc Natl Acad Sci U S A 104:8125-8130.

Zubieta JK, Bueller JA, Jackson LR, Scott DJ, Xu Y, Koeppe RA, Nichols TE, Stohler CS (2005) Placebo effects mediated by endogenous opioid activity on $\mu$-opioid receptors. J Neurosci 25:7754-7762. 